УДК 681.5

\title{
Parametric Synthesis Model Controller for Nonlinear System Modal Control Method
}

\author{
Andrey P. Prokopev*, \\ Vladimir I. Ivanchura* and Rurik T. Emelyanov \\ Siberian Federal University \\ 79 Svobodny, Krasnoyarsk, 660041, Russia
}

Received 03.02.2017, received in revised form 10.02.2017, accepted 15.05.2017

The methods of synthesis of nonlinear PID control system. As a theoretical basis modal synthesis technique used method. The ratios connecting overshoot and settling time of automatic control system with its zeroes and poles arrangement have been obtained. The technique of synthesis of PID control parameters was developed on the basis of these ratios. The numerical illustration is given.

Keywords: automatic control system, the synthesis of PID control, non-linear system, the modal method, overshoot, settling time, zeros and poles of system.

Citation: Prokopev A.P., Ivanchura V.I., Emelyanov R.T. Parametric synthesis model controller for nonlinear system modal control method, J. Sib. Fed. Univ. Eng. technol., 2017, 10(4), 497-507. DOI: 10.17516/1999-494X-2017-10-4-497-507.

(C) Siberian Federal University. All rights reserved

* Corresponding author E-mail address: prok1@yandex.ru, ivan43ura@yandex.ru 


\title{
Параметрический синтез модели регулятора
}

\section{нелинейной системы управления модальным методом}

\author{
А.П. Прокопьев, \\ В.И. Иванчура, Р.Т. Емельянов \\ Сибирский федеральный университет \\ Россия, 660041, Красноярск, пр. Свободный, 79
}

Рассмотрена методика синтеза ПИД-регулятора нелинейной системы управления. В качестве теоретической основы методики синтеза использован модальный метод. Получены соотношения, связывающие перерегулирование и время регулирования системь автоматического регулирования с расположением её нулей и полюсов. На основе этих соотношений разработана методика синтеза параметров ПИД-регулятора. Приводится числовой пример.

Ключевые слова: система автоматического управления, синтез ПИД-регулятора, нелинейная система, модальный метод, перерегулирование, время регулирования, нули и полюсы системы.

\section{Введение}

Наибольшее распространение в автоматизированных системах управления технологическими процессами получили пропорционально-интегрально-дифференцирующие (ПИД) регуляторы.

Проблема синтеза регуляторов систем управления - одна из основных предметных задач теории автоматического управления. В области ПИД-регулирования уже более 60 лет активно ведутся исследования. Было опубликовано огромное количество статей и отчетов, а также книг, в которых обоснована необходимость различных методов синтеза и приведены простые правила расчета параметров ПИД-регуляторов. Известны различные методы параметрического синтеза ПИД-регуляторов $[1,2]$.

Одним из эффективных методов с учетом накопленного практического опыта проектирования и эксплуатации систем управления является модальный метод. Существуют проблемы синтеза регуляторов реальных систем управления мобильными машинами, характеризующимися нелинейностью динамических процессов.

Цель исследования - разработка методики параметрического синтеза ПИД-регулятора нелинейной системы управления с применением модального метода.

\section{Постановка задачи}

Объект исследования - система управления динамическим объектом с обратной связью, представленная как скаляр (одномерная система - Single-Input-Single-Output, SISO).

Изучим вначале процедуру синтеза параметров ПИД-регулятора с передаточной функцией $W_{y}(s)$ в линейной системе

$$
\begin{aligned}
W_{y}(s)=K_{p}+\frac{K_{i}}{s}+K_{d} \cdot s & =\frac{K_{d} \cdot s^{2}+K_{p} \cdot s+K_{i}}{s} . \\
- & 498-
\end{aligned}
$$


К нелинейным системам относят все системы, которые не могут быть описаны линейными дифференциальными уравнениями. В настоящей работе рассматриваются системы управления гидравлическим приводом, который относится к группе естественных нелинейных элементов, присутствующих в автоматических СУ.

Типь нелинейностей. К существенным нелинейностям гидропривода относятся зона нечувствительности, люфты, насыщение по расходу и давлению, сухое (контактное) трение в гидродвигателе [3].

Принцип построения динамической нелинейной структуры зависит как от типа существенной нелинейной характеристики, так и от типа динамического процесса, который предполагается исследовать.

Наиболее эффективным инженерным методом построения нелинейной динамической структуры является метод «обогащения» линейной модели гидропривода теми или иными нелинейными звеньями, обусловленными существенными нелинейностями.

В этом случае нелинейную динамическую структуру можно разделить на две части: линейную (линейную динамическую модель) и нелинейную, которая формируется на основе учитываемой нелинейности. Такой подход позволяет сравнительно просто применить метод гармонической линеаризации и исследовать устойчивость следящей системы с помощью логарифмических частотных характеристик [3].

Ограничение энергетических возможностей насосной установки - давления рабочей жидкости и производительности насоса - приводит к нелинейности типа «насыщение» или «ограничение», выходных сигналов гидропривода. При увеличении амплитуды колебаний золотника выше некоторого значения рост амплитуды первых гармоник выходных сигналов начинает замедляться, а дальнейшее увеличение мощности выходных сигналов происходит за счет возрастания уровня гармонических составляющих более высокого порядка [4].

В системе MATLAB\&Simulink имеется возможность ввода нелинейностей различного вида, которые позволяют описать процессы, не поддающиеся линеаризации. В модели насоса используются нелинейности, которые ограничивают выходную величину.

Блок Saturation (звено с ограничением или насыщением) представляет собой нелинейное устройство, сигнал на выходе которого равен входному сигналу до тех пор, пока не достигает порогов ограничения: верхнего Upper limit или нижнего Lower limit, после этого сигнал перестает изменяться.

Статическая характеристика этого блока приведена на рис. 1 для примера нереверсивного ГП, на рис. 16 - реверсивного ГП.

Структурная схема системы управления с нелинейным звеном типа «ограничение» изображена на рис. 2.

Здесь $W_{o}(s)$ - передаточная функция объекта регулирования; $W_{y}(s)$ - передаточная функция регулятора; $g(s)$ - задающее воздействие; $\varepsilon(s)$ - ошибка регулирования; $u(s)$ - управляющее воздействие; $\bar{u}(s)$ - управляющее воздействие с учетом влияния нелинейного звена; $y(s)$ - выходная регулируемая величина.

Объект регулирования имеет передаточную функцию $W_{o}(s)$ второго порядка

$$
-499-
$$




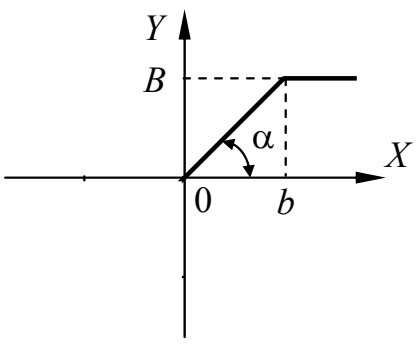

a

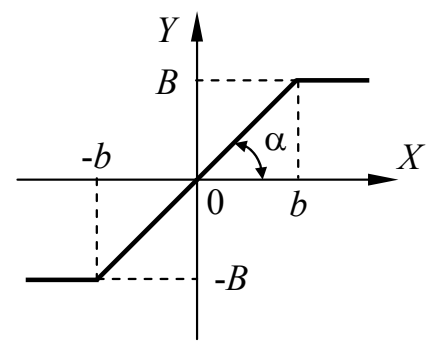

б

Рис. 1. Статическая характеристика блока с ограничением Saturation

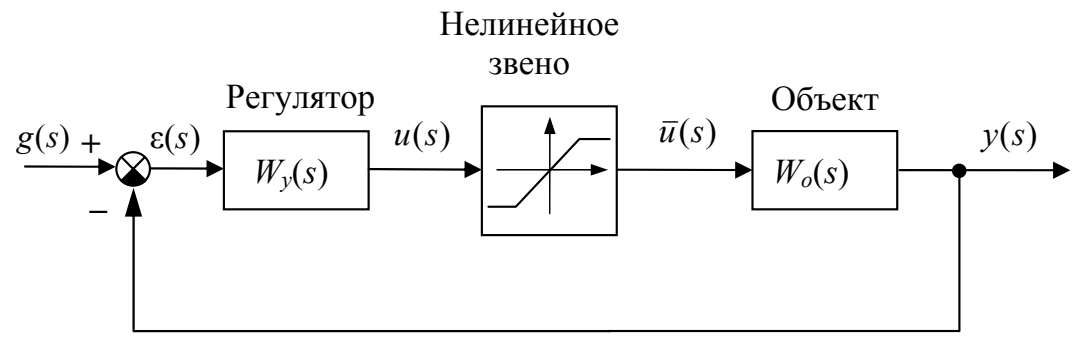

Рис. 2. Структурная схема нелинейной одномерной (SISO) системы управления

$$
W_{o}(s)=\frac{b_{0}}{a_{0} \cdot s^{2}+a_{1} \cdot s+a_{2}} .
$$

Передаточная функция разомкнутого контура с ПИД-регулятором имеет вид

$$
W(s)=W_{y}(s) \cdot W_{o}(s)=\frac{K_{d} \cdot b_{0} \cdot s^{2}+K_{p} \cdot b_{0} \cdot s+K_{i} \cdot b_{0}}{a_{0} \cdot s^{3}+a_{1} \cdot s^{2}+a_{2} \cdot s} .
$$

Определение параметров регулятора

по полюсам передаточной функции замкнутой системь

Полюсы передаточной функции замкнутой системы с ПИД-регулятором в основном определяют характер ее переходной характеристики. Передаточная функция (ПФ) замкнутой системы с ПИД-регулятором в линейном варианте и единичной отрицательной обратной связью в соответствии с выражением (2)

$$
K(s)=\frac{K_{1 d} \cdot b_{0} \cdot s^{2}+K_{1 p} \cdot b_{0} \cdot s+K_{1 i} \cdot b_{0}}{a_{0} \cdot s^{3}+\left(a_{1}+K_{1 d} \cdot b_{0}\right) \cdot s^{2}+\left(a_{2}+K_{1 p} \cdot b_{0}\right) \cdot s+K_{1 i} \cdot b_{0}} .
$$

Характеристическое уравнение замкнутой системы следует из выражения (4):

$$
D(s)=a_{0} \cdot s^{3}+\left(a_{1}+K_{1 d} \cdot b_{0}\right) \cdot s^{2}+\left(a_{2}+K_{1 p} \cdot b_{0}\right) \cdot s+K_{1 i} \cdot b_{0} \cdot
$$

Полюсы ПФ определяют численно по (5) при конкретных значениях $K_{p}, K_{i}, K_{d}$ коэффициентов регулятора. Для системы третьего порядка возможны два варианта полюсов: комплексные и вещественные. 


\section{Комплексные полюсы}

Пусть корни уравнения $D(s)=0$ имеют вид

$$
s_{1}=-\eta_{1}, s_{2}=-\eta_{2}+j \cdot \beta, s_{3}=-\eta_{2}-j \cdot \beta,
$$

где $\eta_{1}$ - степень устойчивости; $\eta_{2}$ - абсолютное значение действительной части полюса; $\beta$ - частота колебаний переходной характеристики, обусловленная полюсами.

И соответствующее характеристическое уравнение примет вид

$$
D_{1}(s)=s^{3}+\left(\eta_{1}+2 \cdot \eta_{2}\right) \cdot s^{2}+\left(\beta^{2}+\eta_{2}^{2}+2 \cdot \eta_{1} \cdot \eta_{2}\right) \cdot s+\eta_{1} \cdot\left(\beta^{2}+\eta_{2}^{2}\right) .
$$

В случае вещественных полюсов

$$
\begin{aligned}
& s_{1}=-\eta_{1}, s_{2}=-\eta_{2}, s_{3}=-\eta_{3} ; \\
& D_{2}(s)=s^{3}+\left(\eta_{1}+\eta_{2}+\eta_{3}\right) \cdot s^{2}+\left[\eta_{3} \cdot\left(\eta_{1}+\eta_{2}\right)+\eta_{1} \cdot \eta_{2}\right] \cdot s+\eta_{1} \cdot \eta_{2} \cdot \eta_{3} .
\end{aligned}
$$

Сравнивая выражения (5), (6) и (5), (7), получаем:

$$
\begin{aligned}
& \frac{a_{1}+K_{1 d} \cdot b_{0}}{a_{0}}=\eta_{1}+2 \cdot \eta_{2} ; \\
& \frac{a_{1}+K_{1 d} \cdot b_{0}}{a_{0}}=\eta_{1}+\eta_{2}+\eta_{3} ; \\
& \frac{a_{2}+K_{1 p} \cdot b_{0}}{a_{0}}=\beta^{2}+\eta_{2}^{2}+2 \cdot \eta_{1} \cdot \eta_{2} ; \\
& \frac{a_{2}+K_{1 p} \cdot b_{0}}{a_{0}}=\eta_{3} \cdot\left(\eta_{1}+\eta_{2}\right)+\eta_{1} \cdot \eta_{2} ; \\
& \frac{K_{1 i} \cdot b_{0}}{a_{0}}=\eta_{1} \cdot\left(\beta^{2}+\eta_{2}^{2}\right) \\
& \frac{K_{1 i} \cdot b_{0}}{a_{0}}=\eta_{1} \cdot \eta_{2} \cdot \eta_{3} \cdot
\end{aligned}
$$

Отсюда находим значения коэффициентов регулятора, выраженные через значения полюсов передаточной функции замкнутой системы с ПИД-регулятором.

В случае комплексных полюсов

$$
\begin{aligned}
& K_{p}=\frac{\left(\beta^{2}+\eta_{2}^{2}+2 \cdot \eta_{1} \cdot \eta_{2}\right) \cdot a_{0}-a_{2}}{b_{0}} ; \\
& K_{i}=\frac{\eta_{1} \cdot\left(\beta^{2}+\eta_{2}^{2}\right) \cdot a_{0}}{b_{0}} ; \\
& K_{d}=\frac{\left(\eta_{1}+2 \cdot \eta_{2}\right) \cdot a_{0}-a_{1}}{b_{0}} .
\end{aligned}
$$

В случае вещественных полюсов 


$$
\begin{aligned}
& K_{p}=\frac{\left[\eta_{3} \cdot\left(\eta_{1}+\eta_{2}\right)+\eta_{1} \cdot \eta_{2}\right] \cdot a_{0}-a_{2}}{b_{0}} ; \\
& K_{i}=\frac{\left(\eta_{1} \cdot \eta_{2} \cdot \eta_{3}\right) \cdot a_{0}}{b_{0}} ; \\
& K_{d}=\frac{\left(\eta_{1}+\eta_{2}+\eta_{3}\right) \cdot a_{0}-a_{1}}{b_{0}}
\end{aligned}
$$

Из выражений (8) и (9) следует очевидный вывод о том, что искомые коэффициенты ПИДрегулятора определяются как параметрами корректируемого объекта в соответствии с выражением (2), так и полюсами передаточной функции замкнутой системы с ПИД-регулятором.

Выражения (8) и (9) позволяют определить параметры ПИД-регулятора при заданном распределении полюсов. Задача определения параметров может быть решена таким образом, если сделать предположение, что введение нелинейного звена между регулятором и объектом управления не изменяет выражений (8) и (9). Следует также учитывать известные [5, 6] сведения по влиянию размещения полюсов на вид переходной характеристики и сведения о влиянии размещения полюсов на связь временных и частотных показателей качества синтезируемой системы, хотя бы без учета нелинейности, возможного запаздывания в системе и изменения ее параметров. Ниже приведена методика параметрического синтеза параметров регулятора в среде программирования Mathcad и MATLAB\&Simulink для случая комплексного размещения полюсов.

\section{Задание на синтез ПИД-регулятора}

1. Введем безразмерные коэффициенты, определяющие соотношение в размещении полюсов. Распределение полюсов при известном значении $\eta_{1}$ определяется коэффициентами $k_{\eta}$ и $k_{\beta}$ :

$$
\eta_{2}\left(k_{\eta}\right)=k_{\eta} \cdot \eta_{1} ; \beta\left(k_{\beta}\right)=k_{\beta} \cdot \eta_{1}
$$

Выбор конкретных значений этих коэффициентов определяется степенью влияния комплексных полюсов на время и вид переходного процесса. Комплексные полюса с целью обеспечения малого перерегулирования необходимо располагать дальше от мнимой оси и вещественного полюса.

2. Определение коэффициентов ПИД-регулятора

$$
\begin{aligned}
& K_{p}\left(k_{\eta}, k_{\beta}, \eta_{1}\right)=\frac{a_{0}}{b_{0}} \cdot\left[\eta_{1}^{2} \cdot\left[\left(k_{\beta}\right)^{2}+\left(k_{\eta}\right)^{2}+2 \cdot k_{\eta}\right]\right]-\frac{a_{2}}{b_{0}} ; \\
& K_{i}\left(k_{\eta}, k_{\beta}, \eta_{1}\right)=\frac{a_{0}}{b_{0}} \cdot \eta_{1}^{3}\left[\left(k_{\beta}\right)^{2}+\left(k_{\eta}\right)^{2}\right] ; \\
& K_{d}\left(k_{\eta}, \eta_{1}\right)=\frac{a_{0}}{b_{0}} \cdot \eta_{1} \cdot\left(1+2 \cdot k_{\eta}\right)-\frac{a_{1}}{b_{0}} .
\end{aligned}
$$

Зададим параметры распределения на основе данных из практики синтеза регуляторов и получим значения коэффициентов ПИД-регуляторов:

$$
\begin{gathered}
K_{p}(30,6,0.5)=5.118 ; K_{i}(30,6,0.5)=2.515 ; K_{d}(30,0.5)=0.564 . \\
-502-
\end{gathered}
$$


Для вещественных корней

$$
\eta_{2}\left(k_{\eta}\right)=k_{\eta} \cdot \eta_{1} ; \eta_{3}\left(k_{\eta}\right)=k_{\eta} \cdot \eta_{1} ; \beta\left(k_{\beta}\right)=k_{\beta} \cdot \eta_{1},
$$

получим коэффициенты ПИД-регулятора

$$
\begin{aligned}
& K_{p}\left(k_{1 \eta}, k_{2 \beta}, \eta_{1}\right)=\frac{\left[\eta_{3} \cdot\left(\eta_{1}+\eta_{2}\right)+\eta_{1} \cdot \eta_{2}\right] \cdot a_{0}-a_{2}}{b_{0}} ; \\
& K_{i}=\frac{\left(\eta_{1} \cdot \eta_{2} \cdot \eta_{3}\right) \cdot a_{0}}{b_{0}} ; \\
& K_{d}=\frac{\left(\eta_{1}+\eta_{2}+\eta_{3}\right) \cdot a_{0}-a_{1}}{b_{0}} .
\end{aligned}
$$

Принимаем вещественные полюса:

$$
\begin{aligned}
& K_{p}\left(k_{1 \eta}, k_{2 \eta}, \eta_{1}\right)=\eta_{1}^{2} \cdot\left(k_{1 \eta}+k_{2 \eta}+k_{1 \eta} \cdot k_{2 \eta}\right) \cdot \frac{a_{0}}{b_{0}}-\frac{a_{2}}{b_{0}} ; \\
& K_{i}\left(k_{1 \eta}, k_{2 \eta}, \eta_{1}\right)=\eta_{1}^{3} \cdot\left(k_{1 \eta} \cdot k_{2 \eta}\right) \cdot \frac{a_{0}}{b_{0}} ; \\
& K_{d}\left(k_{1 \eta}, k_{2 \eta}, \eta_{1}\right)=\eta_{1} \cdot\left(1+k_{1 \eta}+k_{2 \eta}\right) \cdot \frac{a_{0}}{b_{0}}-\frac{a_{1}}{b_{0}} .
\end{aligned}
$$

Пример. Зададим параметры распределения на основе данных из практики проектирования регуляторов и получим значения коэффициентов ПИД-регуляторов:

$$
\begin{aligned}
& K_{p}(30,30,0.451)=3.963 ; \\
& K_{i}(30,30,0.451)=1.775 ; \\
& K_{d}(30,30,0.451)=0.5 .
\end{aligned}
$$

Квадратичная интегральная оценка качества переходного процесса

$$
J_{20}\left(d_{1}(0.5), d_{2}(3.963), d_{3}(1.775)\right)=0.022 .
$$

Определение полюсов в системе с ПИД-регулятором:

$$
\begin{aligned}
& V_{c}(3.963,1.775,0.5)=\left(\begin{array}{c}
204,214 \\
482,923 \\
68,025 \\
2,473
\end{array}\right) ; \\
& \text { polyroots }\left(V_{c}(3.963,1.775,0.5)\right)=\left(\begin{array}{c}
-13,528+0,26 \cdot i \\
-13,528-0,26 \cdot i \\
-0,451
\end{array}\right) .
\end{aligned}
$$

Определение показателей качества переходного процесса и запасов устойчивости. Вычисление квадратичной интегральной оценки $J_{20}$ 


$$
\begin{aligned}
& c_{0}=a_{0}, c_{1}=a_{1}, c_{2}=a_{2}, \\
& d_{0}=a_{0}, d_{1}=a_{1}+K_{d} \cdot b_{0} \\
& J_{20}\left(d_{1}, d_{2}, d_{3}\right)=\frac{c_{0}^{2} \cdot d_{3} \cdot d_{2}+\left(c_{1}^{2}-2 \cdot c_{0} \cdot c_{2}\right) \cdot d_{3} \cdot d_{0}+c_{2}^{2} \cdot d_{1} \cdot d_{0}}{2 \cdot d_{0} \cdot d_{3} \cdot\left(d_{1} \cdot d_{2}-d_{0} \cdot d_{3}\right)} \\
& d_{1}\left(K_{d}\right)=a_{1}+K_{d} \cdot b_{0}, d_{2}\left(K_{p}\right)=a_{2}+K_{p} \cdot b_{0}, d_{3}\left(K_{i}\right)=K_{i} \cdot b_{0} \\
& J_{20}\left(d_{1}\left(K_{d}\right), d_{2}\left(K_{p}\right), d_{3}\left(K_{i}\right)\right)= \\
& =\frac{c_{0}^{2} \cdot K_{i} \cdot b_{0} \cdot\left(a_{2}+K_{p} \cdot b_{0}\right)+\left(c_{1}^{2}-2 \cdot c_{0} \cdot c_{2}\right) \cdot K_{i} \cdot b_{0}+c_{2}^{2} \cdot\left(a_{1}+K_{d} \cdot b_{0}\right) \cdot d_{0}}{2 \cdot d_{0} \cdot K_{i} \cdot b_{0} \cdot\left(\left(a_{1}+K_{d} \cdot b_{0}\right) \cdot\left(a_{2}+K_{p} \cdot b_{0}\right)-d_{0} \cdot K_{i} \cdot b_{0}\right)} .
\end{aligned}
$$

Определение полюсов в системе с ПИД-регулятором:

$$
\begin{aligned}
& V_{c}\left(K_{p}, K_{i}, K_{d}\right)=\left(\begin{array}{c}
K_{i} \cdot b_{0} \\
a_{2}+K_{p} \cdot b_{0} \\
a_{1}+K_{d} \cdot b_{0} \\
a_{0}
\end{array}\right) ; \\
& \text { polyroots }\left(V_{c}\left(K_{p}, K_{i}, K_{d}\right)\right) .
\end{aligned}
$$

Изменение распределения полюсов в комплексной плоскости в случае неудовлетворительных результатов.

Пример. Синтез параметров регулятора при комплексном характере полюсов.

Задание. Определить параметры ПИД-регулятора в системе управления скоростью движения дорожного катка. Обеспечить время переходного процесса $t_{\mathrm{p}}<0,5$, перерегулирование $\sigma \%<5 \%$.

\section{Методика синтеза}

1. Распределение полюсов:

$$
\eta_{1}=\left(t_{p}+\frac{a_{1}}{b_{0}}\right) \cdot \frac{b_{0}}{a_{0} \cdot\left(1+\left(k_{1 \eta}+k_{2 \eta}\right)\right)}=\left(0,5+\frac{a_{1}}{b_{0}}\right) \cdot \frac{b_{0}}{a_{0} \cdot(1+(30+30))}=0,451 .
$$

2. Определение коэффициентов ПИД-регулятора:

$$
K_{p}(30,6,0.451)=4,12 ; K_{i}(30,6,0.451)=1,846 ; K_{d}(30,0.451)=0,5 .
$$

3. Вычисление квадратичной интегральной оценки $J_{20}$ :

$$
J_{20}\left(d_{1}(0.5), d_{2}(4.12), d_{3}(0.5)\right)=0,031 .
$$

4. Определение полюсов в системе с ПИД-регулятором:

$$
V_{c}(4.12,1.846,0.5)=\left(\begin{array}{c}
212,382 \\
500,986 \\
68,025 \\
2,473
\end{array}\right)
$$




$$
\operatorname{polyroots}\left(V_{c}(4.12,1.846,0.5)\right)=\left(\begin{array}{c}
-13,528-2,715 \cdot i \\
-13,528+2,715 \cdot i \\
-0,451
\end{array}\right) \text {. }
$$

Полюсы замкнутой линейной системы при найденных значениях параметров регулятора соответствуют заданному распределению.

5. Определение запасов устойчивости в системе с ПИД-регулятором для вычисленных значений коэффициентов ПИД-регулятора:

$$
K_{p}=4,12 ; K_{i}=1,846 ; K_{d}=0,5 .
$$

Передаточная функция разомкнутой системы без учета нелинейности

$$
W(s)=\frac{K_{d} \cdot b_{0} \cdot s^{2}+K_{p} \cdot b_{0} \cdot s+K_{i} \cdot b_{0}}{a_{0} \cdot s^{3}+a_{1} \cdot s^{2}+a_{2} \cdot s} .
$$

Определяем частоту среза $\omega_{\mathrm{cp}}=a$ и запасы устойчивости в радианах $\gamma$ и градусах $\gamma_{1}$ : начальное значение частоты $\omega=10$; дано $|W(j \omega)|=1$; $a=\operatorname{Find}(\omega)$, получим частоту среза $a=24,48$.

Запас устойчивости $\gamma=\pi+\arg (W(j \cdot a))$, тогда запас устойчивости по фазе в радианах $\gamma=1,42$ и в градусах $\gamma_{1}=\gamma \cdot \frac{180}{\pi}=1,419 \cdot \frac{180}{\pi}=81,31$.

Таким образом, запас устойчивости по фазе составил 81.31 градуса, что указывает на большой запас устойчивости системы и переходная характеристика системы имеет практически апериодический характер изменения (перерегулирование $\sigma \%<2 \%$ ).

6. Определение вида переходной характеристики и других временных функций по имитационной модели (рис. 3).

Переходная характеристика с синтезированными параметрами ПИД-регулятора показана на рис. 4 (параметры ПИД-регулятора: $K_{p}=4,12 ; K_{i}=1,846 ; K_{d}=0,5 ; J_{20}=0,031$ ).

Важно также отметить, что апериодический характер процесса наиболее благоприятен для гидравлических приводов строительных и дорожных машин, работающих в условиях значительных динамических нагрузок - уменьшается вероятность гидравлических ударов.

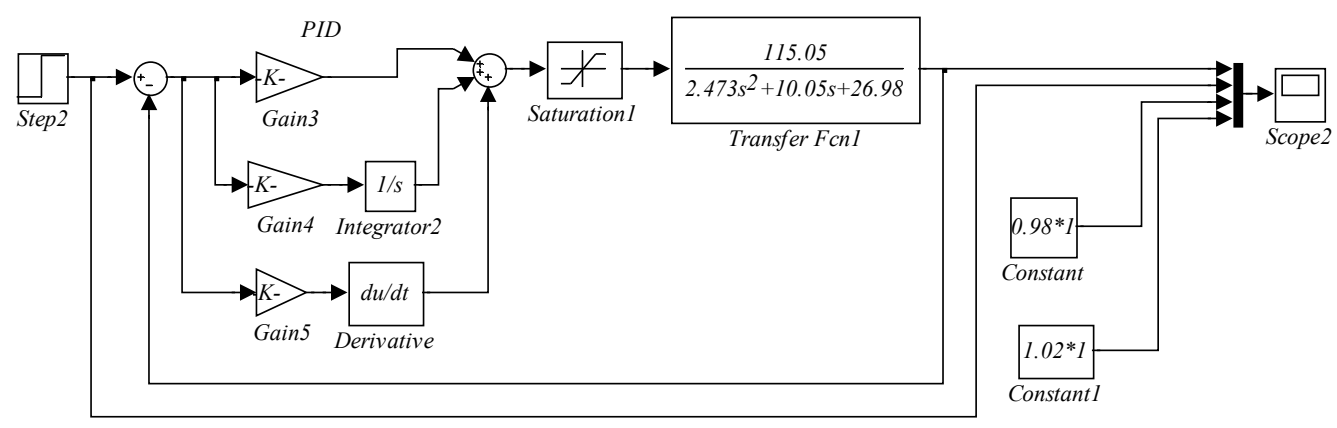

Рис. 3. Имитационная модель нелинейной системы с ПИД-регулятором на языке Simulink

$$
-505-
$$




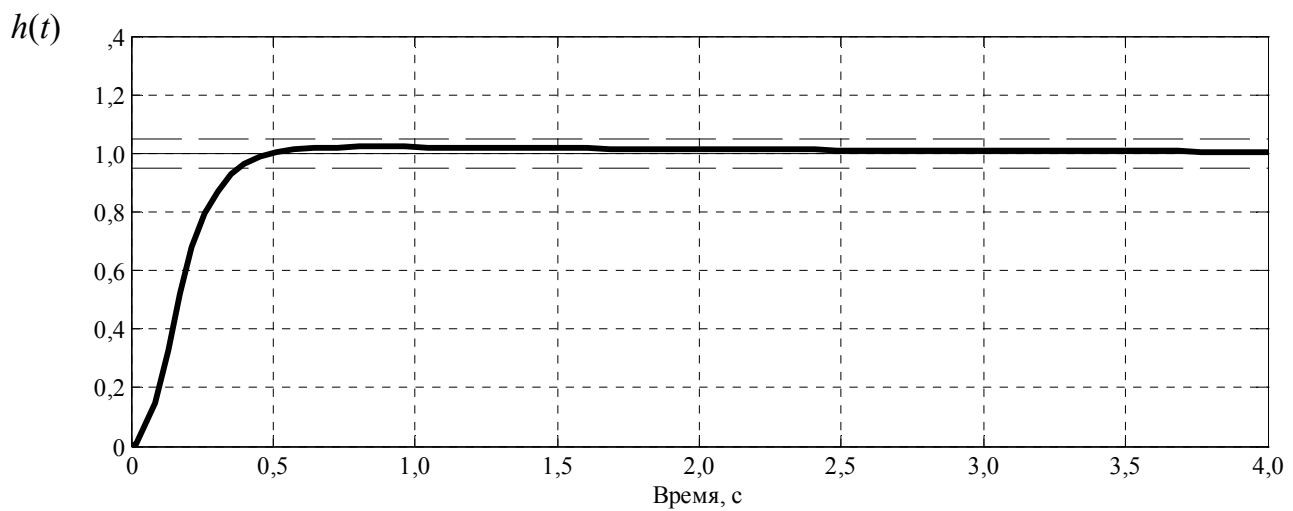

Рис. 4. Переходная характеристика с синтезированными параметрами регулятора (время переходного процесса $t_{\mathrm{p}}=0,4 \mathrm{c}$, перерегулирование $\sigma \%<2 \%$ )

Таким образом, параметры ПИД-регулятора в системе управления с гидравлическим приводом - нелинейным звеном типа «ограничение», должны выбираться так, чтобы в соответствующей линейной системе практически отсутствовало перерегулирование.

\section{Заключение}

На основе модального метода разработана методика синтеза ПИД-регулятора нелинейной системы управления. Получены соотношения, связывающие перерегулирование и время регулирования системы автоматического регулирования с расположением её нулей и полюсов. Определено, что параметры ПИД-регулятора в системе управления с гидравлическим приводом - нелинейным звеном типа «ограничение», должны выбираться так, чтобы в соответствующей линейной системе практически отсутствовало перерегулирование.

\section{Список литературы}

[1] Александров А.Г., Паленов М.В. Состояние и перспективы развития адаптивных ПИДрегуляторов. Автомат. и телемех., 2014, 2, 16-30 [Alexandrov A.G., Palenov M.V. Adaptive PID Controllers: State of the Art and Development Prospects, Automation and remote control, 2014, 2, 16-30 (in Russian)]

[2] Alexandrov A.G., Palenov M.V. Adaptive PID controllers: State of the art and development prospects, Automation and Remote Control, 2014, 75(2), 188-199.

[3] Гамынин Н.С. Гидравлический привод систем управления. М.: Машиностроение, 1972. 376 c. [Gamynin N.S. Gidravlicheskij privod sistem upravlenija. M.: Mashinostroenie, 1972.376 p. (in Russian)]

[4] Циммерман В.В. Нелинейные свойства электрогидравлического вибрационного источника сейсмических колебаний. Проблемы нелинейной сейсмики. ИФЗ АН СССР. М.: Наука, 1987. С. 273-279. Режим доступа: http://www.seisel.com/docs/i_nelsv.pdf. [Cimmerman V.V. Nelinejnye svojstva jelektrogidravlicheskogo vibracionnogo istochnika sejsmicheskih kolebanij. Problemy nelinejnoj sejsmiki. IFZ AN SSSR. M.: Nauka, 1987. P. 273-279. (in Russian)]

$$
-506-
$$


[5] Вадутов О.С. Синтез ПИД-регулятора в системах с запаздыванием методом условной оптимизации с ограничениями на размещение полюсов. Известия Томского политехн. ун-та, 2014, 325(5), 1622. [Vadutov O.S. Design of PID controller for delayed systems using optimization technique under pole assignment constraints, Bulletin of the Tomsk Polytechnic University, 2014, 325(5), 1622 (in Russian)]

[6] Кан Б.А., Кривошеев В.П. Параметрический синтез дискретного алгоритма ПИДрегулятора частотным методом. Информатика и системы управления, 2013, 3(37), 143-151 [Kan B.A., Krivosheev V.P. Parametric synthesis algorithm of discrete PID controller frequency response method, Informatics and control systems, 2013, 3(37), 143-151 (in Russian)] 